\title{
THE RESPIRATORY DEAD SPACE MEASURED BY SINGLE BREATH ANALYSIS OF OXYGEN, CARBON DIOXIDE, NITROGEN OR HELIUM ${ }^{1}$
}

\author{
By J. BARTELS,2 J. W. SEVERINGHAUS, R. E. FORSTER, W. A. BRISCOE, AND \\ D. V. BATES \\ (From the Department of Physiology and Pharmacology, Graduate School of Medicine, Uni- \\ versity of Pennsylvania, and the Department of Anesthesiology, Hospital of the \\ University of Pennsylvania, Philadelphia, Pa.)
}

(Submitted for publication August 3, 1953; accepted September 2, 1953)

Fowler (1) estimated the respiratory dead space in man by recording simultaneously and continuously the nitrogen concentration and the volume flow at the mouth during a single expiration, following a breath of oxygen. Such an expiration may be divided into three phases. In the first part of expiration there is virtually no nitrogen. This is followed by an S-shaped rise in nitrogen concentration as alveolar gas begins to appear. Finally, when the dead space is washed out, the third phase, the 'alveolar plateau' is recorded. By substituting for the S-shaped curve a vertical line which represents a theoretical square front between dead space air and alveolar air, and computing the expired volume up to this front, Fowler obtained an estimate of the dead space for nitrogen, defined as "the volume of the conducting airway down to the location at which a large change in gas composition occurs."

The respiratory dead space for oxygen and carbon dioxide is of interest for several reasons. Firstly, the dead space presumably includes upper airways, bronchi and bronchioles. It is possible that there may be an appreciable transfer of oxygen and carbon dioxide between gas and blood in the smaller bronchioles, though not for the relatively insoluble gas nitrogen; in this case, these fine tubes would no longer be "dead space" for oxygen and carbon dioxide, so that the dead space volume for these gases would be smaller than for nitrogen. DuBois, Fowler, Soffer, and Fenn (2) measured the respiratory dead space for carbon dioxide but did not make comparisons of it with that for other gases in their subjects. Pappenheimer, Fishman, and Borrero (3), using an en-

1 This work was supported in part by the National Heart Institute, United States Public Health Service, Grant H-406(C3).

2 Medical Research Fellow of the National Tuberculosis Association. tirely different approach, recently showed that the dead space for carbon dioxide was the same as that for oxygen. They also made no comparisons with other gases.

Secondly, Krogh and Lindhard (4) and Douglas and Haldane (5), making measurements upon "alveolar" carbon dioxide, obtained vastly different results for the respiratory dead space. Haldane believed that a representative alveolar sample could only be obtained at the end of a forced expiration, and that samples collected earlier in expiration were contaminated by dead space gas. Krogh and Lindhard showed that in the later stages of a single expiration, the concentration of carbon dioxide, when plotted against time, rose to a plateau. They believed that an expired volume of about three times the dead space was sufficient to wash out the dead space completely and that subsequent changes in concentration were due to variations in the composition of alveolar gas itself. Fowler (6) confirmed the approximate straight line characteristic of the 'alveolar plateau' when he analyzed nitrogen continuously in the expired air during a single breath. He showed that once the nitrogen concentration curve reached the alveolar plateau, the changes in concentration occurring thereafter, if any, were due to uneven ventilation and not to further mixing of alveolar gas with dead space gas. The availability of a mass spectrometer for rapid gas analysis enabled us to make measurements of the alveolar plateau and of the dead space for oxygen, carbon dioxide, nitrogen and helium. With the possible exception of the helium dead space in some subjects, we have been able to demonstrate not only that the various dead spaces are the same, but also that the same volume of expired gas is required to completely wash out the dead space for oxygen, carbon dioxide or nitrogen. 


\section{METHODS}

A. Instruments. To study the changing concentration of various gases present in a breath during expiration, we used a mass spectrometer ${ }^{3}$ which continually samples any stream of gases that is led past it, by drawing $8 \mathrm{ml}$. of gas per minute through a metal tube of 0.006 inch bore. The output was led through a DC amplifier to a magnetic pen writer. There was a latency interval of 0.06 second due primarily to the time needed for the gas to traverse the sampling tube and enter the analyzer. Another 0.06 second was then required for 90 per cent response, resulting from the delay in the electrometer circuit and washout of the various chambers of the instrument. The analytic error of the system was less than 1 per cent of full scale deflection on the record. The output was linear, and concentrations of less than 0.05 per cent oxygen, 0.04 per cent carbon dioxide, 0.05 per cent nitrogen and 0.25 per cent helium could be detected. The resolving power and other characteristics of the instrument made the output independent of other gases present in the sample, within the limits required by these experiments.

In a number of experiments, we used a Lilly nitrogen meter as well as the mass spectrometer, to obtain simultaneous and continuous records of expired carbon dioxide, or oxygen, with nitrogen. The accuracy of this meter was \pm 2 per cent of full scale deflection. It had a latency interval of 0.06 second, required another 0.03 second for 90 per cent response and sampled at the rate of $12 \mathrm{ml}$. per minute.

It was necessary to make accurate measurements of volume flow for only approximately $400 \mathrm{ml}$. of expired gas. Therefore we used a 1 liter spirometer with a $70 \mathrm{Gm}$. bell. To the shaft of the pulley was attached a 10,000 ohm wire wound low torque potentiometer 4 with a $11 / 2$ volt battery across it. The contact voltage was led through a DC amplifier to a magnetic pen writer. The pen deflection was a linear function of spirometer bell displacement, and gave a continuous record of the first $400 \mathrm{ml}$. of expired gas. The system was accurate within 1 per cent of full scale at flow rates up to at least 115 liters per minute. The 90 per cent response time was less than 0.02 second.

Initially, we used a Lilly flow meter to estimate expired volume. However, the integrations were laborious, and in addition, the Lilly flow meter was affected by condensation of water on the screen to such an extent that its sensitivity to flow was appreciably changed by the water vapor in one breath. Although this effect could have been corrected, it was simpler to use the spirometer which is not influenced by water vapor, and which yields a volume record that does not require further calculations.

Inspired volume was recorded on an ordinary 6 liter spirometer which was filled with air, 100 per cent oxygen,

${ }^{8}$ Consolidated Engineering Corporation, Pasadena, California.

4 Helipot Company, South Pasadena, California.

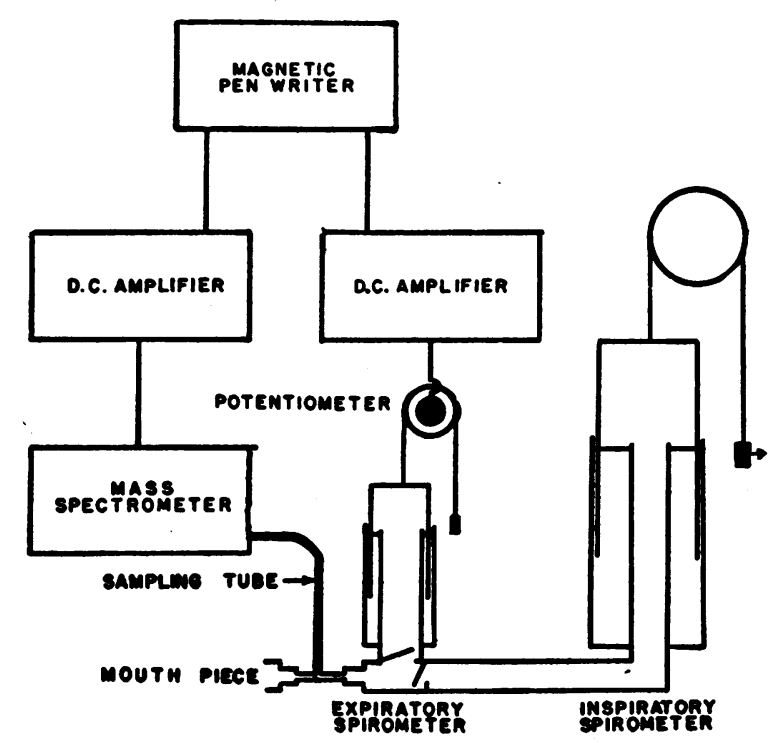

Fig. 1. Arrangement of Apparatus

80 per cent helium-20 per cent oxygen, 100 per cent nitrogen or 100 per cent nitrous oxide, depending on the gas we wished to study during expiration.

The arrangement of apparatus is shown in Figure 1. The sampling tubes of the mass spectrometer and of the nitrogen meter were fitted in the mouth piece. The instrumental dead space, from the edge of the mouthpiece to the tip of the sampling tubes, was $9 \mathrm{ml}$. In order to obtain an average sample from the gas stream, we found it necessary from experiments on model systems, to constrict the lumen of the mouthpiece, stepwise, to about $6 \mathrm{~mm}$. diameter at the site of connection with the sampling tubes. At the expiratory flow rates used, the pressure across this constriction was $0.5 \mathrm{~cm}$. of $\mathrm{H}_{2} \mathrm{O}$ or less. We routinely made a correction of 0.09 second for the lag of the mass spectrometer, regardless of the speed of concentration change, because we found from the same experiments on models that this single correction figure combined convenience and accuracy within the limits of error of the method.

$B$. Subjects. All were research workers, trained to

TABLE I

Physical characteristics of subjects

\begin{tabular}{cccc}
\hline \hline Subject & $\begin{array}{c}\text { Age } \\
\text { (years) }\end{array}$ & $\begin{array}{c}\text { Height } \\
\text { (feet and inches) }\end{array}$ & $\begin{array}{c}\text { Weight } \\
\text { (lbs.) }\end{array}$ \\
\hline 1 & 34 & $5-6$ & 140 \\
2 & 32 & $6-2$ & 165 \\
3 & 30 & $5-10$ & 145 \\
4 & 33 & $5-10$ & 180 \\
5 & 31 & $6-2$ & 185 \\
6 & 34 & $5-11$ & 160 \\
7 & 27 & $5-7$ & 150 \\
8 & 33 & $6-4$ & 200 \\
9 & 35 & $5-11$ & 150 \\
10 & 35 & $6-5$ & 190
\end{tabular}




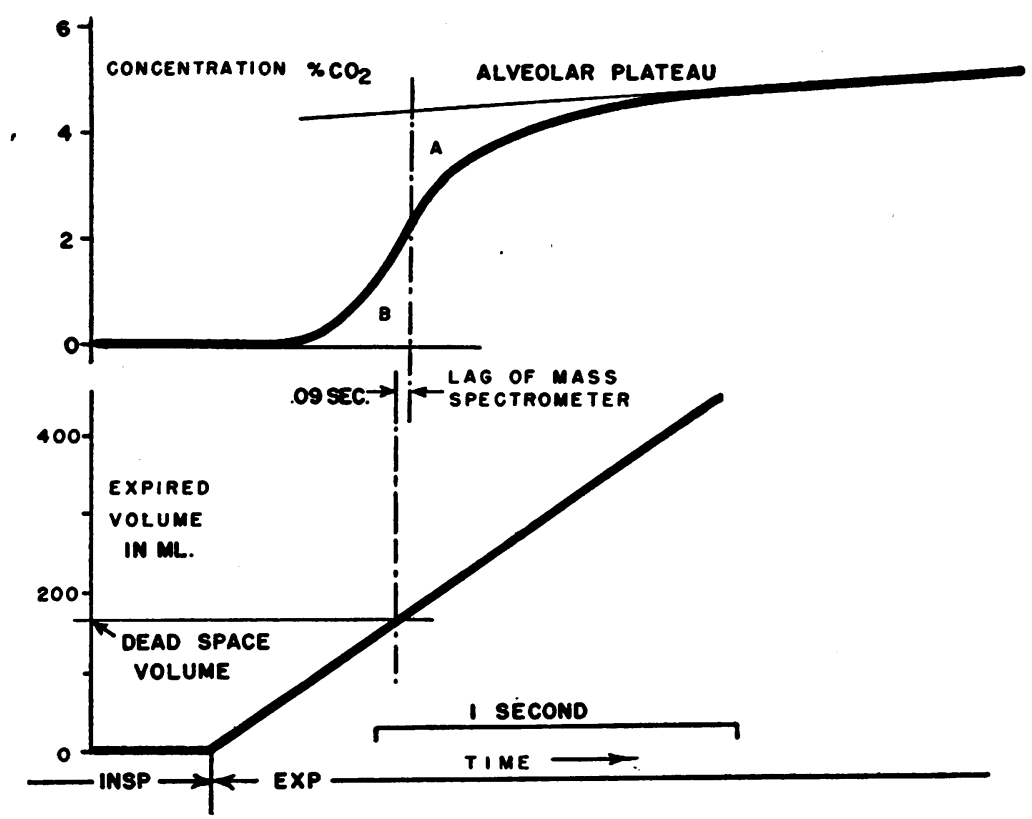

FIGURE 2

In this schematic representation, the upper part of the record is from the mass spectrometer, the lower part from the light-weight one liter spirometer with attached potentiometer. A straight line is drawn through the 'alveolar plateau.' A vertical line is then drawn so that area A equals area $B$. This shows the time at which the transition from dead space gas to alveolar gas would have occurred if the alveolar gas had come through the mouth as a square front. As we were interested in the first part of the expired breath only, the gain of the potentiometer recording system was turned up so high that after about $400 \mathrm{ml}$. were expired, the pen writer ran off scale.

control their breathing. Their physical measurements are given in Table I. Subject 1 was a woman, the others were men. Subject 1 had a therapeutic unilateral pneumothorax about eight years prior to these experiments. Subject 10 had a pleural effusion twelve years prior to these experiments. Otherwise, all subjects were in good health.

$C$. Procedures. The subject always sat in the same position and wore a noseclip. From his normal end-expiratory level, he took a breath of 300 to $900 \mathrm{ml}$. from the 6 liter spirometer. After holding his breath with closed glottis for periods ranging from 1 to 60 seconds, counted from beginning of inspiration to beginning of expiration, he expired evenly into the 1 liter spirometer. Breathholding times of 5 seconds or less were measured to the nearest 0.04 second on the mass spectrometer record. Longer breathholding times were measured with a stopwatch. A continuous record of gas concentration and of the first part of the volume flow was made during this expiration. Although only the first $400 \mathrm{ml}$. were graphically recorded, the subject expired more than 1 liter at a steady rate, the excess escaping around the spirometer bell.

\section{RESULTS}

Figure 2 shows schematically a typical record of expired gas concentration and volume. The theoretical boundary separating dead space and alveoli was found by Fowler's 'estimation' technique (1) which assumes that although the flow rate varies markedly during expiration it does not change greatly during the fraction of a second when the gas concentration is changing most rapidly. The inaccuracy introduced by this technique is less than the other errors of measurement. After correcting for time lag of the mass spectrometer, the amount of gas which has passed the sampling tip of the mass spectrometer up to the theoretical boundary is determined by dropping a perpendicular line to the volume record. From this value, $9 \mathrm{ml}$. is subtracted (dead space of mouthpiece) and the resulting figure, corrected to 


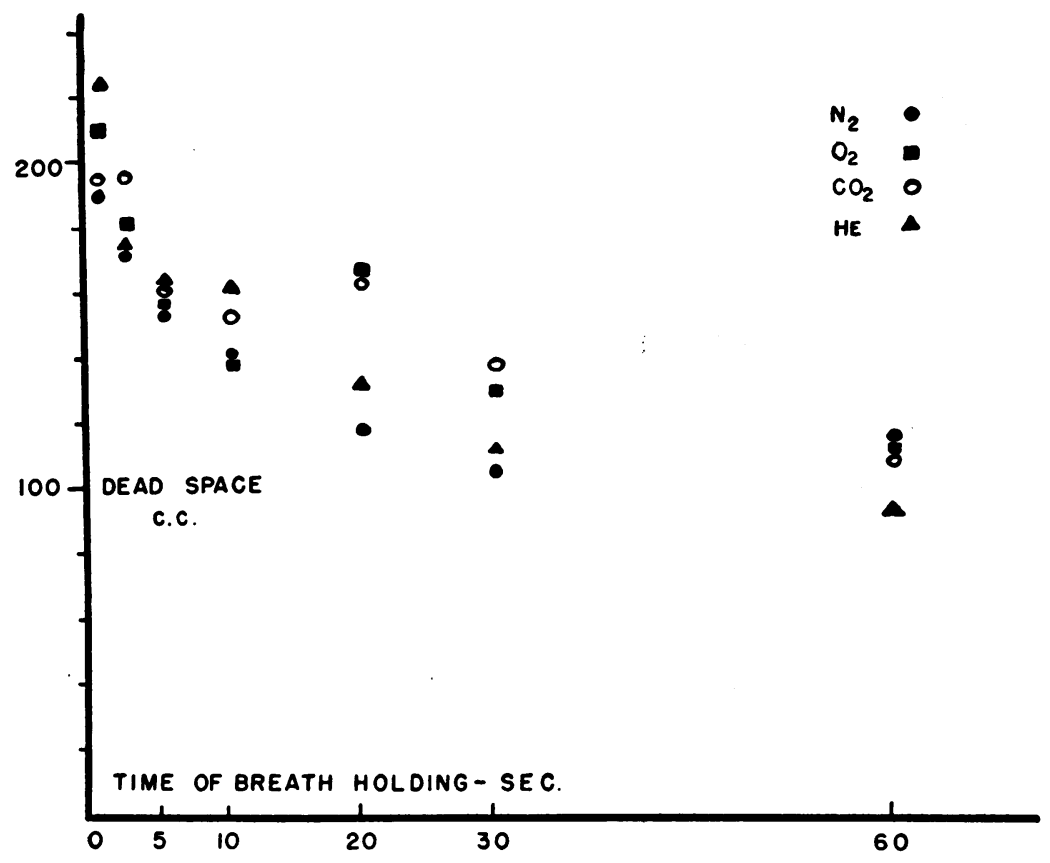

Fig. 3. Decrease of One Subject's Dead Space Volume Versus Time of Breath holding for Four Different Indicator Gases

All inspirations were 80 per cent helium-20 per cent oxygen.

BTPS, is the dead space volume for the indicator gas in question.

The data for Subject 3 are shown in Figure 3; the volume of the dead space is plotted against the time of breathholding, for four different indicator gases. Each point is the average of 2 to 10 observations. It is evident that the dead space for all four indicator gases decreases with increasing time of breathholding in the same manner. We do not feel that the unusually large dead space volumes for oxygen and carbon dioxide after 20 seconds of breathholding, seen only in this subject during this experiment, have any special significance. The standard error of any point on the graph was as high as $\pm 20 \mathrm{ml}$. With the exception noted above, this curve was typical for those subjects $(2,3,4,6$, and 10) in whom similar observations were carried out. There was no significant difference in the rate of decrease of dead space volume with time of breathholding among the different indicator gases.

The scatter of the points seemed to be the same for the various times of breathholding. In an effort to reduce this scatter, we standardized the conditions of the experiment more rigidly, and made a large number of observations on Subjects $1,5,6,9$, and 10 , at $2 \frac{1}{2}$ seconds of breathholding for all four indicator gases: oxygen, carbon dioxide, nitrogen, and helium. The inspired gas was 80 per cent helium-20 per cent oxygen throughout. The subjects grasped the mouthpiece, which consisted of a metal tube, with their lips only, breathing through clenched teeth. This detail was necessary because we observed that allowing the teeth to be separated could produce an increase in dead space of 50 to $100 \mathrm{ml}$. as shown by Lindhard (7). Table II shows that there were no consistent differences in volume of dead space in Subjects 6, 9,

TABLE II

Dead space (ml.) of five subjects for four gases*

\begin{tabular}{ccccc}
\hline \hline Subject & $\mathrm{O}_{2}$ & $\mathrm{CO}_{2}$ & $\mathrm{~N}_{2}$ & $\mathrm{He}$ \\
\hline 1 & $161 \pm 8$ & $144 \pm 4$ & $134 \pm 6$ & $124 \pm 5$ \\
5 & $149 \pm 4$ & $145 \pm 4$ & $141 \pm 5$ & $128 \pm 3$ \\
6 & $169 \pm 9$ & $161 \pm 6$ & $152 \pm 4$ & $165 \pm 3$ \\
9 & $165 \pm 5$ & $160 \pm 5$ & $155 \pm 6$ & $169 \pm 3$ \\
10 & $195 \pm 5$ & $207 \pm 7$ & $198 \pm 9$ & $189 \pm 5$
\end{tabular}

* All breathholding times were about $2 \frac{1}{2}$ seconds. All inspirations were 80 per cent helium-20 per cent oxygen, and followed a period of breathing air. The figures are averages and standard errors of the mean of 3 to $20 \mathrm{ob}-$ servations, and are corrected to BTPS. Subjects had their teeth clenched. 
and 10, for the four indicator gases used. The differences shown by Subjects 1 and 5 will be discussed below.

To determine the effect of breathing helium on the oxygen and carbon dioxide dead spaces, we compared in subjects $1,4,5,6,9$, and 10 the dead space of these two gases following: (a) A breath of air; (b) a single breath of 80 per cent helium20 per cent oxygen; and (c) a three minute washout of the alveoli with this same mixture. We found no consistent difference.

In the experiments described so far, the dead space for the four indicator gases had to be measured on different breaths since our mass spectrometer will record only the concentration of one gas at a time. However, if the nitrogen meter is used to analyze for nitrogen while the mass spectrometer analyzes for oxygen or carbon dioxide, it is possible to obtain a continuous record of the concentration of two gases during the same expiration. This method was used on Subjects $5,6,7,8$, and 10 . The subject inspired one breath of either 100 per cent nitrous oxide or 100 per cent oxygen, held his breath for $2 \frac{1}{2}$ to 30 seconds, and expired through a mouthpiece fitted with sampling tubes leading to both the mass spectrometer and a nitrogen meter. One hundred per cent oxygen was inspired when nitrogen and carbon dioxide concentrations were to be measured during expiration, and 100 per cent nitrous oxide when nitrogen and oxygen were to be measured. $\mathrm{Ni}$ trous oxide was selected by elimination. Among the available gases, nitrogen and oxygen could not be used because the dilution by water vapor of a dry inspired gas in the dead space produced a stepwise decrease in the record of the expired gas concentration which confused interpretation, if this same gas was used as the indicator. Helium could not be used because it distorts the $\mathrm{N}_{2}$ meter output.

To compensate for the differences in absolute value and slope of the respective alveolar plateaus, the expired gas concentrations at each point were recalculated as percentages of the extrapolated 'alveolar plateaus' at that point. As shown in Figure 4, the corrected nitrogen concentration from the nitrogen meter record, and the corrected oxygen or carbon dioxide concentration from the mass spectrometer record always described virtually the same curve. For example, the deviation between the two curves presented in Figure 4 corresponded to a difference in dead space of less than $3 \mathrm{ml}$.

\section{DISCUSSION}

Subjects 1 and 5 in Table II had dead spaces for helium which were significantly smaller than those for some of the other gases. However, for reasons beyond our knowledge or control, the variation among a series of dead space determina-

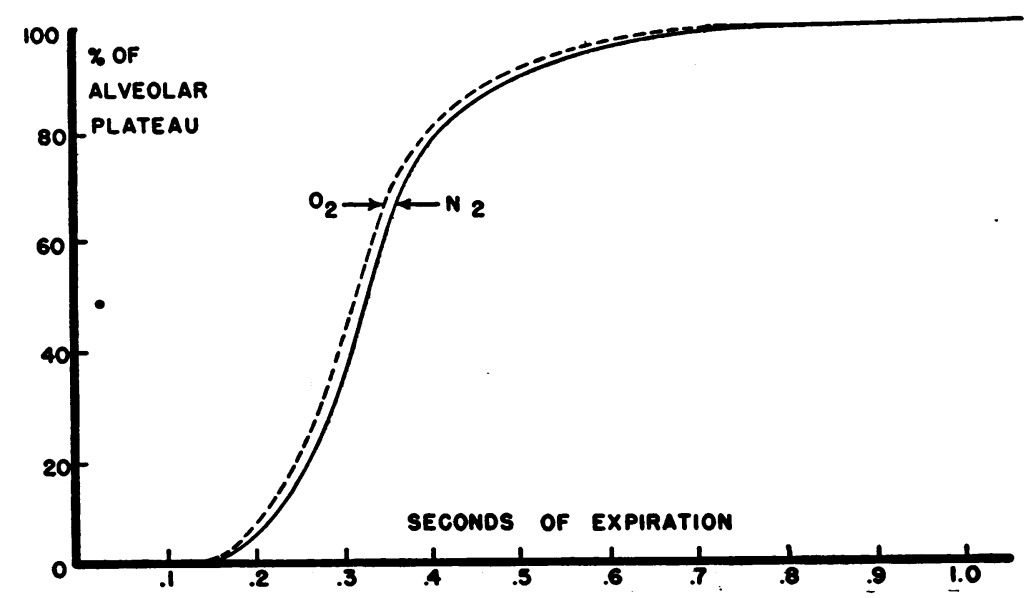

Fig. 4. Simultaneous Recording of Nitrogen Concentration (Nitrogen Meter) and Oxygen Concentration (Mass Spectrometer)

Both concentrations are expressed as percentages of their respective 'alveolar plateaus.' The difference in dead space volume resulting from the above shown difference in concentration is less than $3 \mathrm{ml}$. 
tions on one occasion was not as great as the variation between the mean values of dead space determined on different occasions, in spite of the fact that the position of the subject, lung volume, inspired and alveolar gases, as well as the time of breathholding were apparently the same. For example, under controlled conditions, the carbon dioxide dead space of Subject 9 varied from 147 $\mathrm{ml}$. to $186 \mathrm{ml}$. (unreported data; each the average of five observations) in one afternoon, a significant change. This type of variation occurred to some degree in all subjects. The small unavoidable differences in breathholding time which occurred were not great enough to have produced this variation nor was there a correlation. Therefore, we think that the physiological significance of these dead space differences is uncertain. The simultaneous use of the mass spectrometer and the nitrogen meter proved most useful when comparing the dead space for different gases because this unexplained variation between different breaths was eliminated. Unfortunately, helium could not be studied in this fashion, because it changes the sensitivity of the nitrogen meter. In any case the dead space for helium was not more than about $20 \mathrm{ml}$. smaller than the dead space for the other gases studied.

As stated above, Fowler (6) has shown that in the case of nitrogen in normal subjects by the time the rising concentration joins the 'alveolar plateau,' the expired breath contains pure alveolar gas. Since the $\mathrm{O}_{2}$ and $\mathrm{CO}_{2}$ concentrations in the expired breath, expressed as fractions of their extrapolated 'plateau' values, are the same as the $\mathrm{N}_{2}$ concentration (Figure 4), we have concluded that the dead spaces for $\mathrm{O}_{2}$ and $\mathrm{CO}_{2}$ are also completely washed out by the time their respective concentration curves have joined their 'alveolar plateaus.'

Gas exchange in the dead space could be studied in a precise manner by means of the simultaneous nitrogen and oxygen (or carbon dioxide) records corrected to fractions of their respective alveolar concentrations. During the entire expiration the two curves coincided. There were two interesting exceptions to this generalization. Firstly, in some experiments there was a stepwise change in concentration at the foot of the S-shaped rise (Figure 5). Simultaneous records of nitrogen and oxygen (or carbon dioxide) concentrations in the expired gas demonstrated that the two gases followed the same concentration course during the step in the majority of instances. In those few experiments where this was not true, the nitrogen fraction was as often greater than the oxygen (or carbon dioxide) as vice versa. Therefore, we believe these steps did not represent significant gas exchange in the dead space. Secondly, in the simultaneous nitrogen and oxygen curves of all five subjects the oxygen concentration appeared to rise more rapidly than the nitrogen concentration (Figure 4), in particular after the breath was held for 20 seconds or more. This effect is equivalent to a time difference between the two concentration curves of 0.02 seconds, or to a decrease in $\mathrm{O}_{2}$ dead space of about $2 \mathrm{ml}$. These differences were so small that we are unable to say at present whether they were artefacts or not.

Galdston and Horwitz (8) reported an average rise in carbon dioxide tension and fall in oxygen tension of $3.7 \mathrm{~mm}$. $\mathrm{Hg}$ (about 0.52 per cent of an atmosphere change in carbon dioxide or oxygen concentration) in the supraglottic portion of the airway during periods of breathholding of up to 30 seconds. Forssander (9), who studied carbon dioxide only, showed that this exchange did not take place in the oral cavity. Following, as noted above, an inspiration of 100 per cent oxygen, simultaneous records of carbon dioxide and nitrogen concentrations in the expired gas showed that the carbon dioxide concentration did not rise (less than 0.05 per cent) until the nitrogen concentration started to rise, even after 30 seconds of breathholding. This made it unlikely that there was carbon dioxide exchange in that part of the expired gas that contained no alveolar gas (absolute dead space). The fact that the nitrogen and carbon dioxide concentration curves coincided during the rapid buildup of concentration in the expired gas made it improbable that a significant amount of carbon dioxide had diffused into the dead space. The oxygen concentration in the absolute dead space, under conditions comparable to those of Galdston, was difficult to interpret in terms of gas exchange because of the water vapor dilution mentioned above. As it was unlikely that oxygen would exchange to a greater extent than the far more soluble carbon dioxide, we do not believe that there was a significant exchange of carbon dioxide and oxygen between the dead space and the surrounding tissues. 


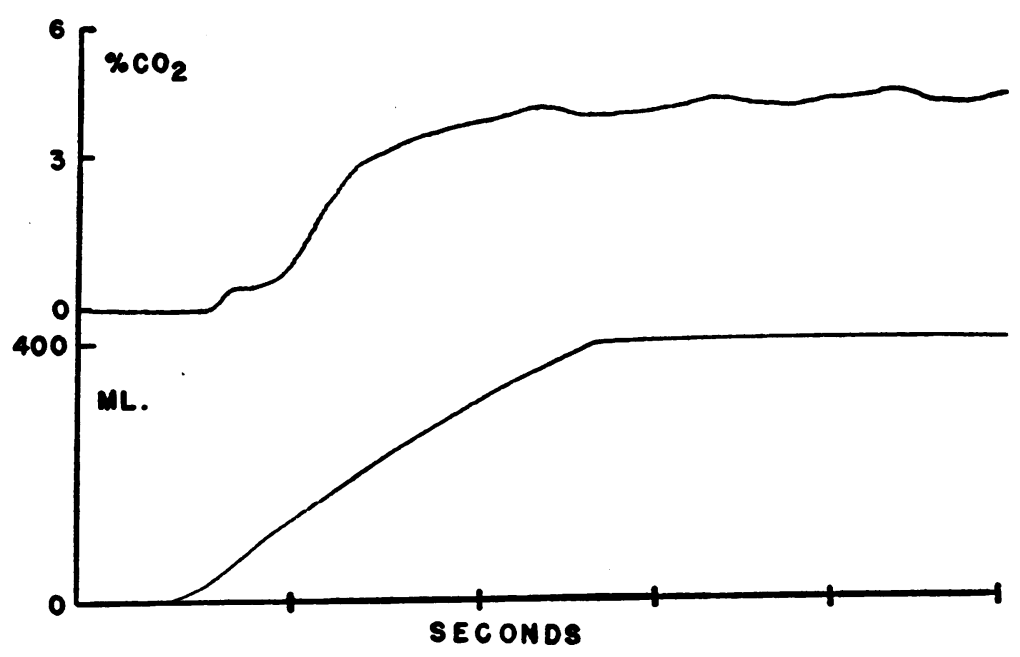

Fig. 5. Reproduction of a Record of Carbon Dioxide Concentration and Volume of Subject 5 during Expiration

The co-ordinates are the same as in Figure 2. The upper line is the carbon dioxide concentration, and shows a 'step' at the foot of the S-curve, as well as cyclic concentration changes synchronous with the heart beat. The lower line is expired gas volume. The terminal horizontal part of this line is produced by the recording pen reaching its limit of movement.

Figure 5 also shows how the shape of a number of curves was apparently affected by the action of the heart. The small changes in concentration synchronous with the heart beat are much more prominent in the records of some subjects than in those of others, for no obvious reason. When present, they continue throughout each entire expiration. This 'cardiac ripple' was most prominent in oxygen and carbon dioxide curves but it was also present in a number of nitrogen and helium curves. We did not observe in any of our records of alveolar oxygen concentration the paradoxical inversion of the alveolar plateau late in expiration described by Cotton (10).

Fowler had shown that the nitrogen dead space decreased with increasing time of breathholding, and DuBois did the same for the carbon dioxide dead space. In Figure 3 we showed this decrease to be the same for oxygen, carbon dioxide and helium as for nitrogen. This decrease cannot be due mainly to diffusion because it is approximately the same for helium as for the other gases. The rapid diffusion of helium should have resulted in a smaller dead space. The most important cause of the decrease with time of the dead space volume is probably the churning action of the heart, as suggested by DuBois. Just why the curve is steep- est for breathholding times up to 5 seconds is not clear. The shape of the curve suggests that at time zero the dead space might be at least twice as large as it is after $2 \frac{1}{2}$ seconds of breathholding. The curve further shows that the rate of decrease after 20 seconds of breathholding becomes very small indeed. This is in contradistinction to one of DuBois' subjects whose dead space was reduced to zero after 55 seconds of breathholding. None of our subjects approached zero values after $60 \mathrm{sec}-$ onds of breathholding. Otherwise, our data for the dead space agree well with those of Fowler, DuBois, Pappenheimer, and Krogh to the extent that our studies were comparable with these.

The very first portion of every expired breath represents pure dead space gas without any admixture of alveolar gas. We found the volume of this 'absolute dead space' to vary considerably from breath to breath, but the simultaneous oxygen or carbon dioxide and nitrogen records showed that on the same breath it was the same for these three gases. We compared the absolute dead space of Subjects 1, 5, 6, 9, and 10 for helium and nitrogen, and found it to vary from 15 to $80 \mathrm{ml}$., but without any trend that would differentiate between these two gases. 


\section{SUM MARY}

1. Using a rapidly recording mass spectrometer and a light weight spirometer with attached potentiometer, the respiratory dead space was estimated with oxygen, carbon dioxide, nitrogen and helium as indicator gases.

2. In five normal subjects ranging in age from 27 to 35 years, the dead space decreased to the same degree with increasing breathholding times for all four indicator gases.

3. The dead space for the four indicator gases was essentially the same in three of five subjects, and in the other two the dead space for helium appeared to be somewhat $(20 \mathrm{ml}$.) smaller than for the other indicator gases. Breathing helium did not decrease the oxygen, carbon dioxide or nitrogen dead spaces.

4. Using the mass spectrometer simultaneously with a Lilly nitrogen meter, expiratory gas concentration curves were obtained in five subjects for oxygen, carbon dioxide, and nitrogen; these were so similar that there was no measurable difference in dead space volume.

\section{REFERENCES}

1. Fowler, W. S., The respiratory dead space. Am. J. Physiol., 1948, 154, 405.
2. DuBois, A. B., Fowler, R. C., Soffer, A., and Fenn, W. O., Alveolar $\mathrm{CO}_{2}$ measured by expiration into the Rapid Infrared Gas Analyzer. J. Appl. Physiol., 1952, 4, 526.

3. Pappenheimer, J. R., Fishman, A. P., and Borrero, L. M., New experimental methods for determination of effective alveolar gas composition and respiratory dead space, in the anesthetized dog and in man. J. Appl. Physiol., 1952, 4, 855.

4. Krogh, A., and Lindhard, J., On the average composition of the alveolar air and its variations during the respiratory cycle. J. Physiol., 1914, 47, 431.

5. Douglas, C. G., and Haldane, J. S., The capacity of the air passages under varying physiological conditions. J. Physiol., 1912, 45, 235.

6. Fowler, W. S., Uneven pulmonary ventilation in normal subjects and in patients with pulmonary disease. J. Appl. Physiol., 1949, 2, 283.

7. Lindhard, J., The "dead space" in breathing. Proc. Physiol. Soc., 1914, J. Physiol., 1914, 48, xliv.

8. Galdston, M., and Horwitz, S. A., Study of the exchange of oxygen and carbon dioxide in the supraglottic portion of the respiratory dead space. Am. J. Physiol., 1948, 155, 420.

9. Forssander, C. A., A study of carbon dioxide present in the oral cavity during inspiration. J. Appl. Physiol., 1949, 2, 175.

10. Cotton, F. S., Studies in respiration. On the occurrence of an apparently paradoxical rise in the oxygen percentage of increasingly deeper samples of alveolar air. Australian J. Exper. Biol. \& M. Sc., 1939, 17, 433. 